\title{
Identification of thrombospondin 1 (TSP-1) as a novel mediator of cell injury in kidney ischemia
}

\author{
Charuhas V. Thakar, ${ }^{1}$ Kamyar Zahedi, ${ }^{2}$ Monica P. Revelo, ${ }^{3}$ Zhaohui Wang, ${ }^{1}$ Charles E. Burnham, ${ }^{1}$ \\ Sharon Barone, ${ }^{1}$ Shannon Bevans, ${ }^{4}$ Alex B. Lentsch, ${ }^{5}$ Hamid Rabb, ${ }^{4}$ and Manoocher Soleimani ${ }^{1,6}$ \\ 'Department of Medicine, University of Cincinnati School of Medicine, Cincinnati, Ohio, USA. ²Department of Pediatrics, Children's Hospital Medical Center, \\ Cincinnati, Ohio, USA. ${ }^{3}$ Department of Pathology, University of Cincinnati School of Medicine, Cincinnati, Ohio, USA. ${ }^{4}$ Department of Medicine, \\ Johns Hopkins University, Baltimore, Maryland, USA. ${ }^{5}$ Department of Surgery, University of Cincinnati School of Medicine, Cincinnati, Ohio, USA. \\ ${ }^{6}$ Veterans Affairs Medical Center, Cincinnati, Ohio, USA.
}

\begin{abstract}
Thrombospondin 1 (TSP-1) is a matricellular protein that inhibits angiogenesis and causes apoptosis in vivo and in vitro in several cancerous cells and tissues. Here we identify TSP-1 as the molecule with the highest induction level at 3 hours of IR injury in rat and mouse kidneys subjected to ischemia/reperfusion (IR) injury using the DNA microarray approach. Northern hybridizations demonstrated that TSP-1 expression was undetectable at baseline, induced at 3 and 12 hours, and returned to baseline levels at 48 hours of reperfusion. Immunocytochemical staining identified the injured proximal tubules as the predominant sites of expression of TSP-1 in IR injury and showed colocalization of TSP-1 with activated caspase-3. Addition of purified TSP-1 to normal kidney proximal tubule cells or cells subjected to ATP depletion in vitro induced injury as demonstrated by cytochrome $c$ immunocytochemical staining and caspase-3 activity. The deleterious role of TSP-1 in ischemic kidney injury was demonstrated directly in TSP-1 null mice, which showed significant protection against IR injury-induced renal failure and tubular damage. We propose that TSP-1 is a novel regulator of ischemic damage in the kidney and may play an important role in the pathophysiology of ischemic kidney failure.
\end{abstract}

\section{Introduction}

Ischemia/reperfusion (IR) injury is the major cause of morbidity and mortality in diseases such as acute renal failure, myocardial infarction, and stroke. Ischemic conditions result in ATP depletion and accumulation of toxic metabolites whereas reperfusion results in the production of reactive oxygen intermediates (1-3). The resulting alteration in cellular metabolism and generation of toxic molecules contribute to tissue damage, which is characterized by the presence of necrotic and apoptotic areas in the affected organs $(1,4,5)$. Despite significant development in our understanding of the pathophysiology of IR injury in kidney, there are no specific therapeutic options for patients except for supportive care.

In order to better define the early pathways activated by ischemic injury, we used a DNA microarray to identify changes in messenger RNA levels, which in many cases are a reliable index of gene expression (6). We chose 3 hours after reperfusion as the earliest time at which changes in gene expression might be reliably detected. We were aware that the most critical changes might be small and transient, reflecting the balance between alternative pathways such as necrosis and apoptosis. The results identified several molecules with very high induction at 3 hours of reperfusion. The highest expression belonged to a molecule called thrombospondin 1 (TSP-1). TSP-1 is known to be transactivated by p53 and causes apoptosis in nonepithelial cells and cancerous tissues (7-9). There are no published reports on the role of TSP-1 in caus-

Nonstandard abbreviations used: EST, expressed sequence tag; IR, ischemia/reperfusion; NRK, normal rat kidney; TSP-1, thrombospondin 1.

Conflict of interest: The authors have declared that no conflict of interest exists.

Citation for this article: J. Clin. Invest. 115:3451-3459 (2005).

doi:10.1172/JCI25461. ing apoptosis in epithelial cells or in IR injury. We present a set of studies that indicate the expression pattern of TSP-1 and its localization in damaged kidney tubules representing its novel association in IR injury. We further demonstrate that the induction of TSP-1 precedes the upregulation of $\mathrm{p} 53$ and that TSP-1 can induce proapoptotic changes in cultured kidney cells. Finally, we demonstrate that TSP-1 null mice are significantly protected against kidney failure and structural damage in IR injury. Our results point to the potential role of TSP-1 as an important novel mediator of injury in kidney ischemia.

\section{Results}

Induction of kidney injury. Induction of kidney injury in this model has been demonstrated by our group as well as others. In brief, serum creatinine is increased, and kidneys show all the parameters of acute ischemic injury, including loss of brush border, tubular lumen dilatation or collapse, cellular vacuolization, loss of cell polarity, and detachment from basement membrane.

DNA microarray. Poly $\left(\mathrm{A}^{+}\right) \mathrm{RNA}$ was extracted from the total RNA isolated from kidney cortices of 3 normal rats and 3 rats subjected to 30 minutes of ischemia and 3 hours of reperfusion. cDNA labeled with ${ }^{33} \mathrm{P}$ was prepared using pooled poly $\left(\mathrm{A}^{+}\right) \mathrm{RNA}$ from each group and then used to probe 2 rat GeneFiltersR microarrays. Data were analyzed using Pathways software. Initial analysis was performed by a $\log / \log$ plot of spot intensities for each gene in sham-operated kidneys vs. kidneys with IR injury (Figure 1). Intensity varied over 4 logs with a slope of 0.9 , perhaps indicating a slight bias toward the sham-operated DNA hybridization, which was performed first. However, because we defined changes of interest as those lying outside the $95 \%$ prediction interval, we do not think this bias affected our ability to detect changes caused by IR 

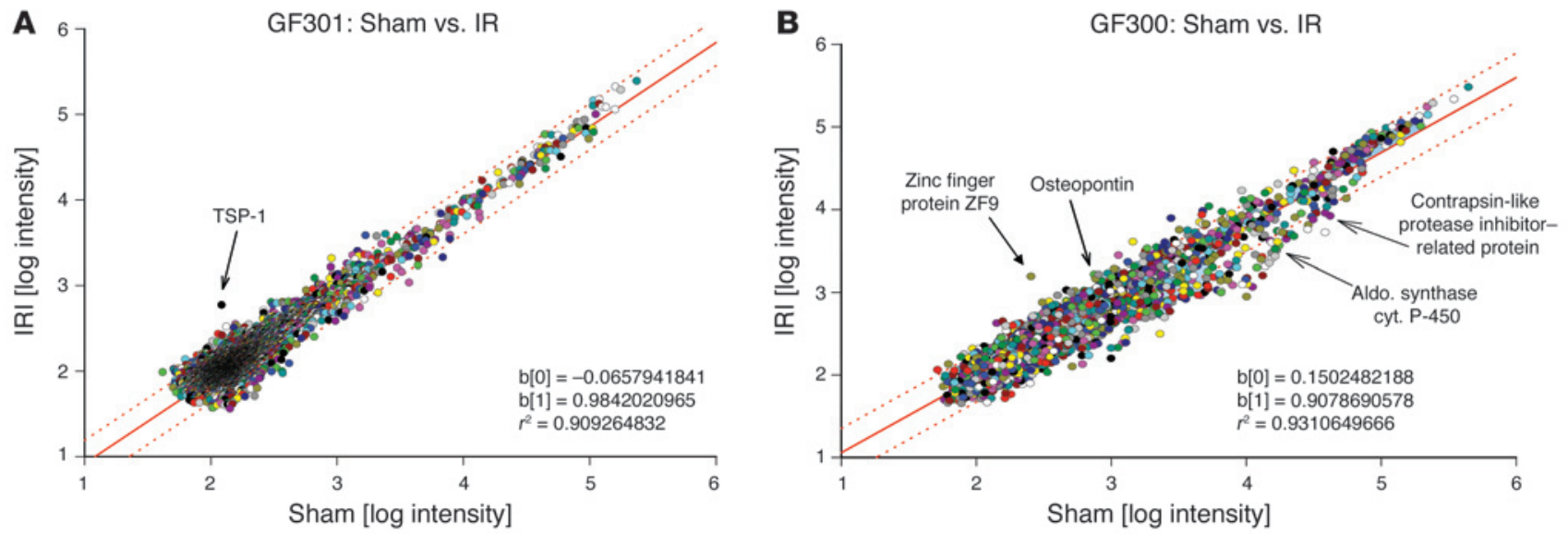

Figure 1

DNA microarray results. Two separate filters, GF301 (A) and GF300 (B), were probed with the ${ }^{33}$ P-labeled cDNA, which was prepared using poly $(\mathrm{A}+)$ RNA from sham-operated kidneys and kidneys with IR injury (IRI). Aldo. synthase, aldosterone synthase; cyt. P-450, cytochrome p450; $\mathrm{b}[0], y$ intercept; $\mathrm{b}[1]$, slope; $r^{2}$, correlation coefficient.

injury. A logarithmic plot of the hybridization intensities showed a small cluster of genes, which was downregulated, and a diffuse area of upregulated genes, making it apparent from the graph that more messages are decreased than increased by IR injury at 3 hours. The 50 largest increases and decreases were selected for analysis using Pathways software and BLAST (http://www.ncbi. nlm.nih.gov/BLAST/) searches of GenBank.

The hybridization intensities appeared to be similar, with more clustering at lower intensities and a slope more nearly equal to 1 . In Figure 1, the majority of the genes indicated are identified and unidentified expressed sequence tags (ESTs). Among the 46 genes displaying the greatest decreases in expression, 30 were ESTs. Most could be unequivocally identified by BLAST analysis. Of the 19 genes with known function, 9 contribute to resistance to apoptosis, including 14-3-3, CAP2, and Spi2. Two are mitochondrial proteins (malate $\mathrm{DH}$ and $\mathrm{BCK} \mathrm{DH}), 2$ protect against oxidative stress (metallothionein and ceruloplasmin), and 1 is a detoxification enzyme (cytosol epoxide hydrolase). The 10 largest decreases in gene expression were of ESTs or genes of unknown function.

Among the 45 genes that displayed the greatest increases in expression on the microarrays, 32 were ESTs. Seven of the 14 genes with known functions fell into 2 groups: growth and repair proteins and vascular proinflammatory genes. Members of the vascular proinflammatory group included genes such as endothelin receptor $\beta$ and plasminogen activator inhibitor I whereas members of the growth and repair family included osteopontin, PDGF- $\alpha$, and casein kinase II.

TSP-1 (Figure 1A) and zinc finger protein 9 (ZF9) (Figure 1B) showed the highest expression among all upregulated genes. Little is known about ZF9, which is a transcription factor known to be upregulated in states associated with fibrosis in liver (10). We examined the expression and properties of TSP-1 in detail, as this molecule is a matricellular protein and has been shown to cause apoptosis, at least in endothelial cells, making it relevant to kidney IR injury.

$m R N A$ expression and immunoblot analysis of TSP-1 in ischemic kidneys. To verify the results of DNA microarray, the expression of TSP-1 was examined in mouse kidney IR injury by Northern hybridization and Western blot analysis. Figure 2A shows a representative
Northern hybridization and indicates the expression of TSP-1 at various reperfusion intervals $(3,12,24$, and 48 hours) following 45 minutes of ischemia. As demonstrated, the expression of TSP-1 was undetectable in sham-operated animals but was heavily induced at 3 hours of reperfusion. The expression of TSP- 1 declined at 12 hours and disappeared by 48 hours after reperfusion. Identical results were obtained with rat kidney (data not shown).

Figure 2B shows a representative Western blot analysis of TSP-1 in kidneys of mice with IR injury. As indicated, TSP-1 abundance was very low in control animals but was significantly upregulated at 3 hours of reperfusion, confirming the results of Northern hybridization experiments. When adjusted for protein loading as determined by $\beta$-actin abundance, the expression of TSP- 1 showed more than an 8 -fold increase in IR injury $(P<0.01, n=3)$.

Figure $2 \mathrm{C}$ (top panel) shows an ethidium bromide staining of RT-PCR on CD36, the receptor that mediates some of the functions of TSP-1, including apoptosis, in several tissues. As demonstrated, CD36 was readily detected in mouse kidney. When examined by Northern hybridization, the expression of CD36 was shown to have increased by $94 \%$ at 3 hours $(P<0.05, n=3)$ and by $48 \%$ at 12 hours $(P<0.05, n=3)$ of reperfusion (Figure $2 \mathrm{C}$, bottom panel).

TSP-1 induction precedes the upregulation of $p 53$ in kidney IR injury. Published reports point to the transactivation of TSP-1 by p53 in cancerous tissues and nonepithelial tissues. There is, however, little information on TSP- 1 in renal injury. To determine the relationship of TSP-1 to p53, the expression of p53 was examined in kidney IR injury by Northern hybridization. Figure 2D shows Northern hybridization and indicates that p53 expression was very low in sham-operated animals at 3 hours of reperfusion but was moderately increased at 12 hours and peaked at 24 hours of reperfusion. The expression of p53 declined significantly at 48 hours after reperfusion. Taken together, these results demonstrate that the induction of TSP-1 precedes the upregulation of p53.

Immunocytochemical staining of TSP-1 in kidney IR injury. To determine the cellular distribution of TSP-1 in kidneys with IR injury, immunofluorescence labeling was performed on sham-operated kidneys and kidneys with IR injury at 3,24, and 48 hours of reperfusion following 45 minutes of ischemia. Immunofluorescence labeling was performed as indicated in Methods. Figure 3 shows 
A

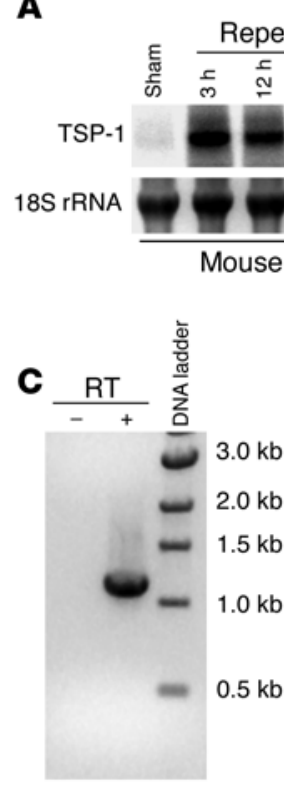

B

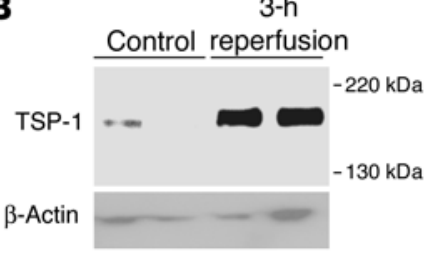

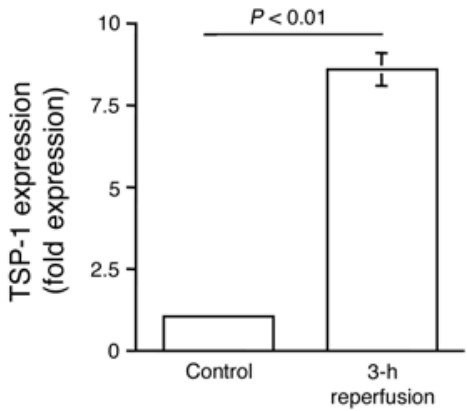

D

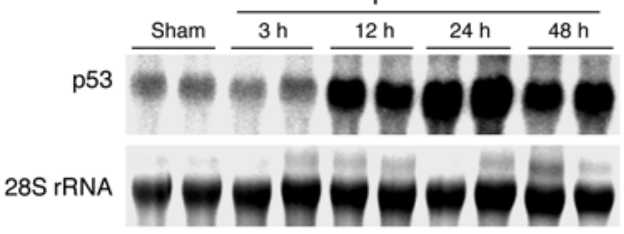

\section{Figure 2}

Examination of the time course of expression of TSP-1 in kidney. (A) TSP-1 expression: Northern hybridization. Northern blot analyses were performed ( $30 \mu \mathrm{g} / \mathrm{well}$ of total RNA) to determine the time course of expression of TSP-1 in IR injury. The expression of TSP-1 was induced at 3 hours of reperfusion, remained elevated at 12 hours of reperfusion, and decreased to undetectable baseline levels at 24 and 48 hours of reperfusion. Equal loading was confirmed by the examination of 18S rRNA bands. (B) TSP-1 expression: immunoblot analysis. Left panel: Western blot analysis was performed as described in Methods. Right panel: quantitation of the results. (C) Expression of CD36 in mouse kidney cortex. Left panel: RT-PCR of CD36. Right panel: Northern hybridization of CD36 in kidney IR injury. (D) Expression of p53 in kidney IR injury. The time course of expression of p53 was examined in IR injury. As indicated, the expression of p53 remained unchanged at 3 hours of reperfusion, increased moderately at 12 hours of reperfusion, and peaked at 24 hours of reperfusion. The expression of p53 decreased significantly at 48 hours of reperfusion. Equal loading was confirmed by the examination of $28 \mathrm{~S}$ rRNA bands.

TSP-1 labeling in sham-operated kidneys (left panel) and kidneys with IR injury at 3 hours of reperfusion (middle and right panels). As indicated, TSP-1 expression in the kidney was absent in sham-operated animals but was readily detectable at 3 hours of reperfusion in several segments, which are predominantly proximal tubules, based on their localization (adjacent to glomeruli), luminal size, and tubular cell morphology. Further, it is apparent that the tubules that expressed TSP-1 were damaged and had collapsed lumen consistent with ischemic injury. These results demonstrate that TSP-1 expression in IR injury is limited to damaged tubules. There was no TSP- 1 expression detected at 24 or 48 hours of reperfusion (data not shown).

Double immunocytochemical staining with TSP-1 and activated caspase-3 in kidney. To determine the status of TSP-1-expressing cells, double immunofluorescence labeling was performed with antibodies against TSP-1 and activated caspase-3, a known marker of cell injury in kidney $(11,12)$. As indicated in Figure 4, TSP-1 (right panels) and activated caspase-3 (left panels) colocalized to the same cells and nephron segments in kidneys with IR injury (merged images). Based on the histology of tubules expressing TSP-1 and the expression of activated caspase- 3 in the same cells, we propose that TSP- 1 expression is detected in cells undergoing programmed cell death. Not all nephron segments expressing TSP-1 expressed activated caspase-3.

Effect of TSP-1 on kidney proximal tubule cells in vitro. In the next series of experiments, the effects of TSP-1 peptides were examined in cultured normal rat kidney (NRK) proximal tubule cells (NRK52 cells). Accordingly, cells were grown to confluence, exposed to 1 $\mu \mathrm{g} / \mathrm{ml}$ TSP- 1 for 24 or 48 hours, and fixed and stained by cytochrome $c$ apoptosis-detection assay. Thereafter, slides were incubated with the nuclear dye Hoechst 33342 to visualize the nuclei. Figure 5, A-D, demonstrates the changes detected in cells treated with TSP-1 versus control cells treated with vehicle at 24 hours. Figure 5A shows punctate granular staining for cytochrome $c$, consistent with its localization to mitochondria. Double labeling with

\section{Figure 3}

Immunofluorescence labeling of TSP-1 in kidney IR injury. Paraffin-embedded kidney sections were examined for the expression of TSP-1 by immunofluorescent microscopy in control (sham operated) and at 3 hours after reperfusion. G, location of glomerulus. Magnification, $\times 400$.
Sham

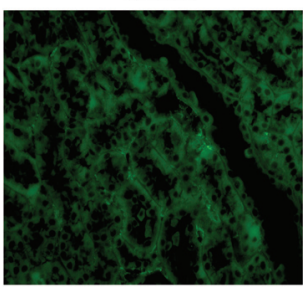

3-h reperfusion

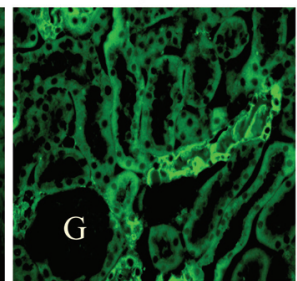

3-h reperfusion

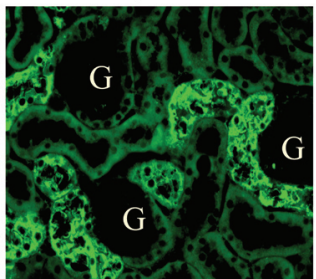



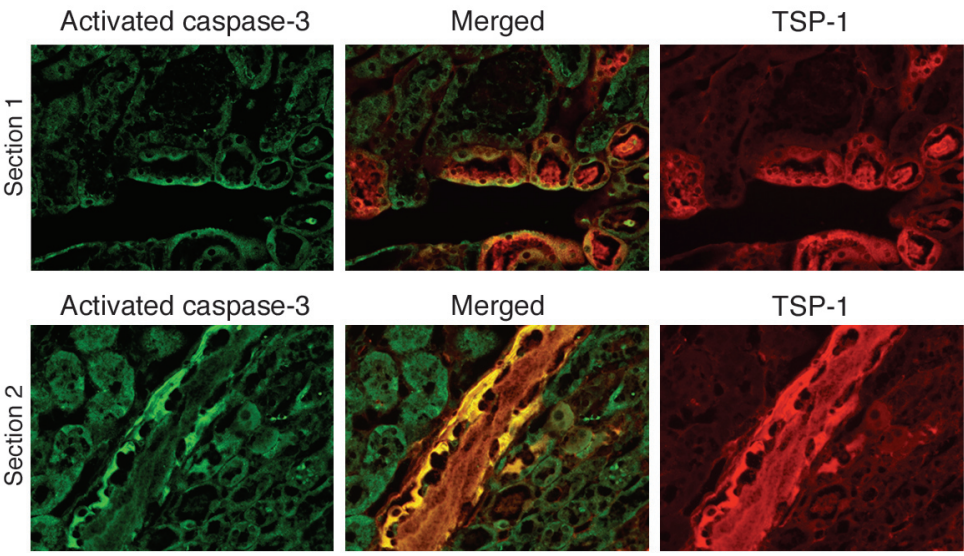

TSP-1

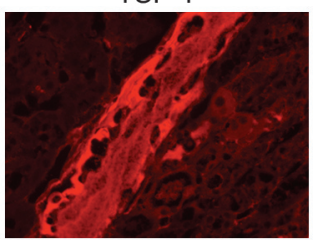

Figure 4

Double immunofluorescence labeling of TSP-1 and activated caspase-3 in kidney IR injury. Shown are 2 separate paraffin-embedded kidney sections from 3-hour reperfusion, examined for the expression of TSP-1 (red) and activated caspase-3 (green) by immunofluorescent microscopy. The merged images (center) indicate the colocalization of the 2 proteins in the same cells. Magnification, $\times 400$.

in normal cells vs. $108 \% \pm 6 \%$ in cells exposed to TSP-1; $P>0.05, n=3$ ). However, cells subjected to oxidative stress (ATP depletion) showed $47 \% \pm 5 \%$ increase in caspase- 3 activity in the presence of TSP- $1(P<0.05, n=3)$.

Effect of TSP-1 gene deletion on kidney function and structure in IR injury. To ascertain the role of TSP-1 in causing nuclear staining is shown in Figure 5B, which also shows intact nuclear membrane. Figure 5, C and D, shows diffuse cytoplasmic staining for cytochrome $\mathrm{c}$ in some cells along with nuclear fragmentation upon double labeling with nuclear stain.

At 48 hours of incubation with TSP-1 (Figure 5, G and H), most cells demonstrated diffuse cytoplasmic staining for cytochrome $c$ in the majority of cells along with extensive nuclear fragmentation upon double labeling. Control vehicle-treated cells remained largely unchanged (Figure 5, E and F). This exemplifies the release of cytochrome $c$ from mitochondria into cytoplasm, consistent with the changes seen in cells undergoing apoptosis (13). Figure 5C shows the caspase- 3 enzymatic activity in kidney proximal tubule (NRK) cells treated with TSP-1 for 24 hours. As demonstrated, caspase- 3 activity increased by more than $3.1 \pm 0.2$-fold in TSP- 1 treated cells as compared with vehicle-treated cells $(P<0.01, n=3$ separate experiments). The results of the above studies demonstrate the induction of significant apoptosis in kidney cells by TSP-1.

The above experiments examined the effect of TSP- 1 on caspase- 3 activation at 24-48 hours. The induction of TSP-1 in kidney IR injury in vivo peaked at 3 hours of reperfusion, well before the time course determined in the above in vitro studies. To ascertain the role of TSP-1 on caspase- 3 activation, temporally comparable to in vivo IR injury, kidney proximal tubule (NRK) cells were subjected to 45 minutes of ATP depletion, an in vitro model of IR injury, in order to recapitulate the in vivo setting of ischemic injury. Thereafter, TSP-1 or diluent was added for 3 hours. The results were compared with those of normal cells incubated with TSP-1 for the same duration. Normal cells showed no significant changes in caspase- 3 activity when exposed to TSP-1 (102\% $\pm 4 \%$

\section{Figure 5}

Effect of TSP-1 on kidney proximal tubule cells in vitro. (A-D) Cytochrome $c$ release at 24 hours. As indicated, immunocytochemical staining was performed for cytochrome $C$ on paraffin-fixed cells. (A and $\mathbf{B}$ ) Vehicle-treated cells showing fine punctate/granular staining for cytochrome $C$ within the cytoplasm (A) and double labeling with nuclear staining (B). (C and $\mathbf{D})$ Same staining procedure performed on cells after incubation with $1 \mathrm{ng} / \mathrm{ml}$ of TSP-1. Magnification, $\times 40$. $(\mathbf{E}-\mathrm{H})$ Cytochrome $c$ release at 48 hours. (E and $\mathbf{F}$ ) Vehicle-treated cells. (G and $\mathbf{H})$ TSP-1-treated cells. Other parameters, including magnification and acquisition of the images were similar to those for A-D. (I) Caspase- 3 activity assay. Caspase- 3 enzymatic activity was measured colorimetrically as described in Methods. As indicated, caspase-3 activity increased significantly in TSP-1-treated cells compared with vehicle-treated cells.

ischemic kidney failure directly, WT and TSP-1 null mice were subjected to kidney IR injury. Accordingly both WT and TSP-1 KO animals underwent 45 minutes of ischemia followed by 24 hours of reperfusion. Measurement of serum creatinine, performed at
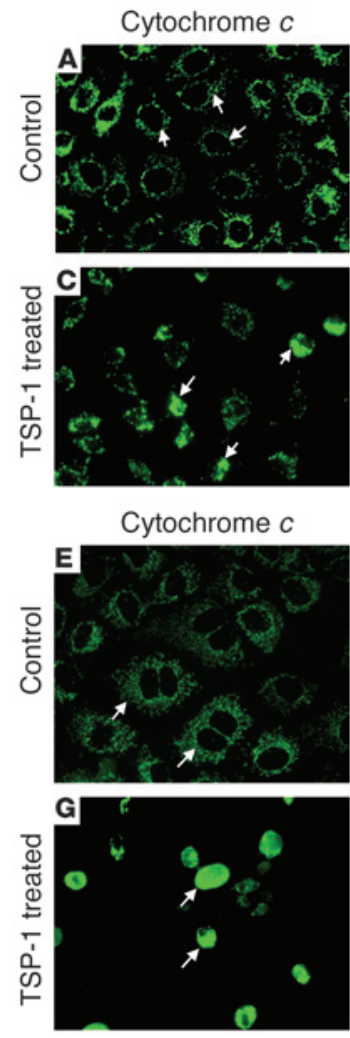

I

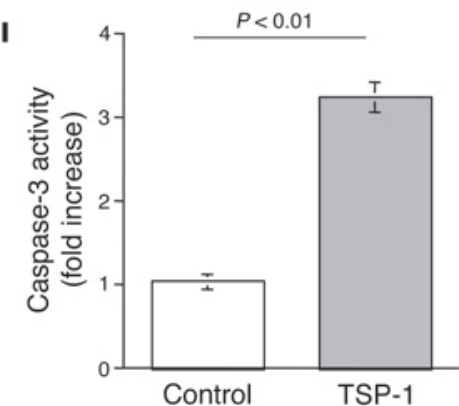

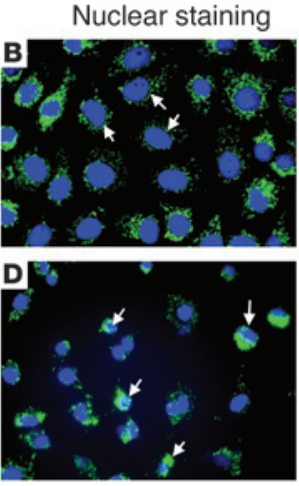
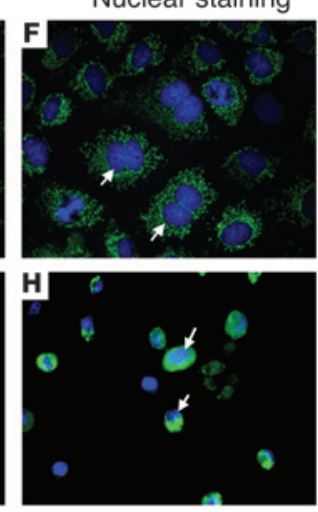
A
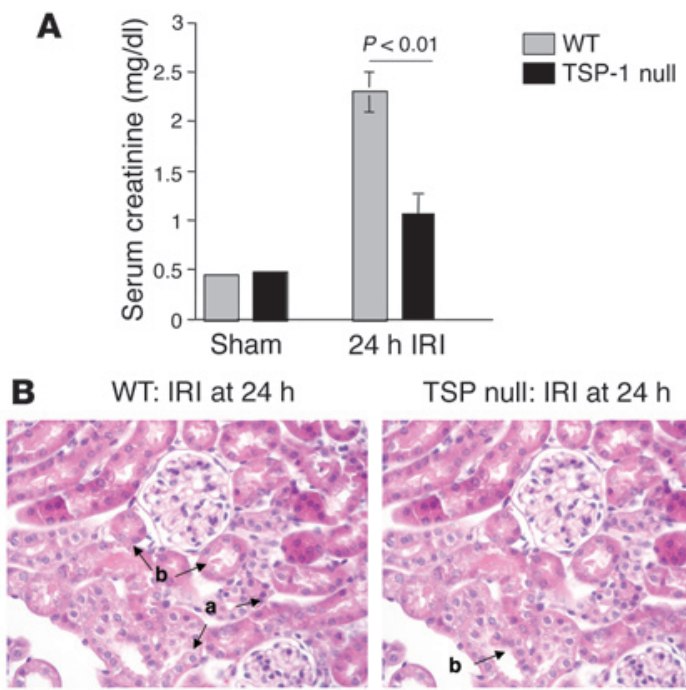

WT: sham operated
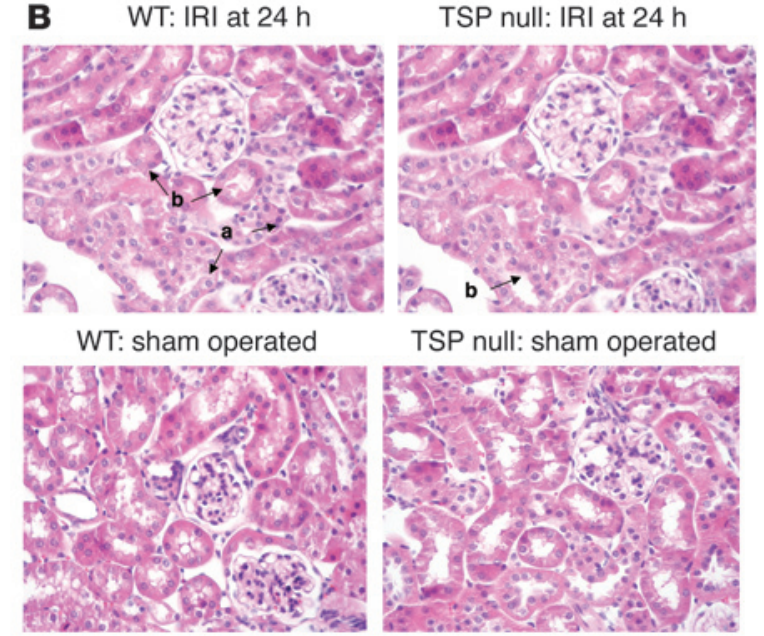

TSP null: sham operated
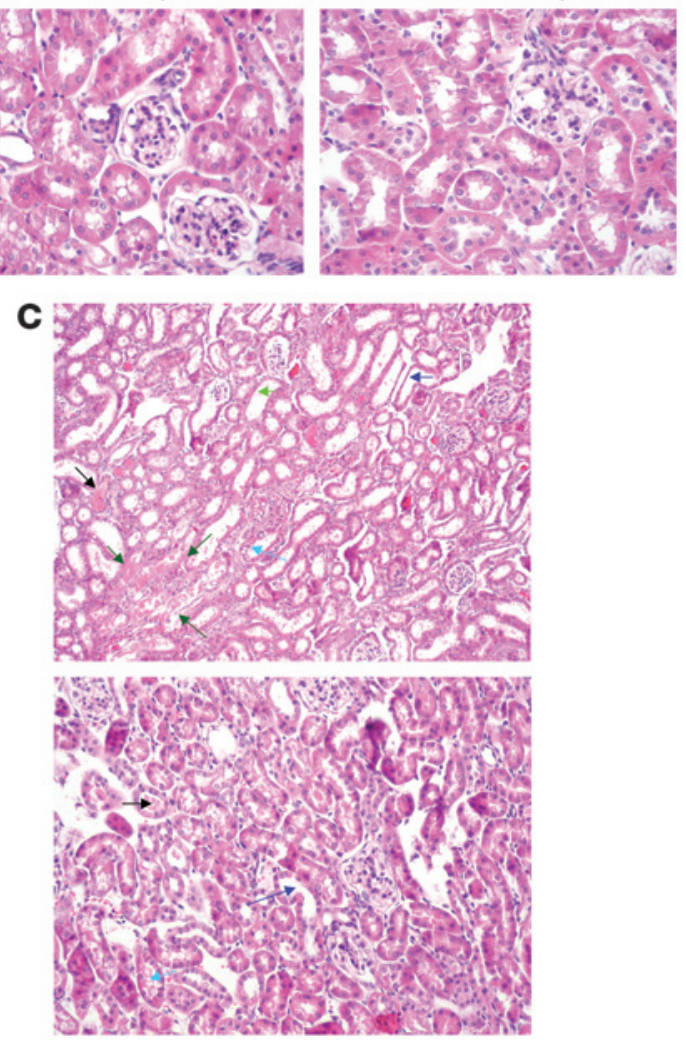

24 hours of reperfusion, is shown in Figure 6A and demonstrates that TSP-1 null mice have significantly lower serum creatinine concentration at 24 hours of reperfusion (2.3 in WT vs. 1.0 in TSP-1 KO animals; $n=5$ in each group, $P<0.01$ ). Comparison of the results demonstrates that the magnitude of renal failure (as determined by rise in serum creatinine levels) was protected by more than $72 \%$ in TSP- 1 null mice (Figure $6 \mathrm{~A}$ ). These results are consistent with significant resistant to acute ischemic renal failure in TSP-1 null mice.

The histological analysis of kidney sections at 24 hours of reperfusion by $\mathrm{H} \& \mathrm{E}$ staining is shown in both low and high magnifications (Figure 6, B and C). Figure 6B (top panel) is a high-magnifi-

\section{Figure 6}

Effect of TSP-1 gene deletion on kidney function and structure in kidney IR injury. (A) Kidney function. As demonstrated, mice deficient in TSP-1 showed significant protection against acute renal failure in IR injury, as assessed by serum creatinine measurement at 24 hours after reperfusion compared with WT animals. (B) Histological analysis of kidneys. Top panel: 24-hour IR injury in WT and TSP-1 null mice. Extensive brush border loss and vacuolization (arrows) were observed in WT animals. Bottom panel: Sham-operated WT and TSP-1 null mice. a, epithelial vacuolization; b, loss of brush border/tubular dilatation. Magnification $\times 40$. (C) Histological analysis of kidneys (low magnification). Top panels: 24-hour IR injury in WT. Bottom panels: TSP-1 null mice. Extensive brush border loss (light green arrows), tubular dilation (dark blue arrows), vacuolization (light blue arrows), cast formation (black arrows), and necrosis (area surrounded by dark green arrows) were observed in WT animals. Magnification $\times 10$.

cation photomicrograph of H\&E-stained kidney sections of WT and TSP-1 null mice and demonstrates significant preservation of tubular integrity in TSP-1 null mice versus WT animals. As shown in Figure 6B (top panel), the kidneys from the WT mice demonstrated severe and widespread injury with extensive loss of brush border, tubular dilation, and vacuolization. In contrast, the kidneys from the TSP-1 null mice demonstrated significant preservation of tubular structures, as manifested by less tubular dilatation, focal vacuolization of the epithelium, and loss of brush border (Figure 6B, top panel). The sham-operated animals for both experimental groups were histologically comparable (Figure $6 \mathrm{~B}$, bottom panel).

Figure 6C (top panel) shows a low-magnification photomicrograph encompassing the entire cortex and shows necrosis, cast formation, tubular dilatation, vacuolization, and brush border loss in WT animals (top panel) at 24 hours of IR injury. The TSP-1 null mice, however, showed remarkable protection against all of the above parameters of injury (Figure 6C, lower panel). The extent of tissue damage in WT and TSP- $1 \mathrm{KO}$ animals was compared in 3 different sections using a scoring matrix described in Methods. The analysis demonstrated that WT animals showed increased loss of brush border (mean score of $3.2 \pm 0.25$ in WT vs. $1.0 \pm 0.15$ in TSP-1 KO mice; $P<0.01)$, cast formation ( $3.3 \pm 0.3$ in WT vs. $2 \pm 0.3$ in TSP- $1 \mathrm{KO}$ mice; $P<0.05)$, necrosis of epithelial cells $(2.4 \pm 0.3$ in WT vs. $0.8 \pm 0.1$ in TSP- $1 \mathrm{KO} ; P<0.01)$, vacuolization ( $3.1 \pm 0.3$ in WT vs. $2 \pm 0.2$ in TSP- $1 \mathrm{KO} ; P<0.05)$ and tubular dilatation $(3.4 \pm 0.25$ in WT vs. $0.9 \pm 0.1$ in TSP- $1 \mathrm{KO}$; $P<0.001)$ in the cortex at 24 hours after IR injury.

With regard to apoptosis, despite the low number of apoptotic cells in both WT and TSP-1 KO mice, a detailed analysis of 30 fields examined from 3 different slides showed that the number of apoptotic tubules was $2.6 \pm 0.2$ per field in WT animals and $1.6 \pm 0.15$ in TSP-1 KO mice at 24 hours of reperfusion $(P<0.01)$.

\section{Discussion}

In clinical settings of IR injury, especially in the kidney, therapeutic options remain extremely limited. Experimental models have shown promise in ameliorating renal injury but only when applied in its early phases (within 24 hours) (14). Thus, it is essential to elucidate further the complex paradigms involved in the pathogenesis of IR injury with a goal to translate to therapeutic targets. The results of current studies utilizing the tool of DNA microarray demonstrate distinct patterns of expression for genes that represent early pathways ( 3 hours after injury) as activated by IR injury 
(Figure 1). TSP-1 was 1 of the 2 genes that showed highest levels of expression, and due to its known function as a proapoptotic protein and a regulator of angiogenesis, we chose to study its role in the setting of IR injury. The results of DNA microarray were verified by demonstrating an increased mRNA expression of TSP-1, which peaked at 3 hours and declined to baseline levels by 48 hours, after kidney IR injury (Figure 2). These events were undetectable in sham-operated animals. The expression of TSP-1 protein followed a similar time course as indicated by Western blotting and immunocytochemical staining and confirmed its predominant localization to the portions of the renal tubules that sustained damage after IR injury (Figures 2, 3, and 4).

To evaluate the role of TSP as a mediator of injury in renal tubular cells, we performed a series of experiments where cultured rat proximal tubule cells (NRK52) were incubated with TSP-1. At 24 and 48 hours of incubation, proapoptotic changes were clearly detected in the cells by cytochrome $c$ assay (Figure 5). Cells undergoing apoptosis released cytochrome $c$ from the mitochondria into cytoplasm, which in turn led to the activation of caspase pathway, leading to cell apoptosis. Changes consistent with these events were demonstrated in NRK cells in which the pattern of cytochrome $c$ staining changed from granular (mitochondrial localization) to diffuse (cytosolic release) along with nuclear fragmentation (13). We interpret these data to indicate that upregulation of TSP-1 in the setting of IR injury may play a direct role as a mediator of tubular injury. The definitive experiments with regard to the deleterious role of TSP-1 in acute ischemic renal failure came from studies in WT and TSP-1 null mice that were subjected to IR injury. These results demonstrated that mice deficient in TSP-1 showed significant protection against renal failure caused by ischemic damage (Figure 6A). The protection against IR injury-induced renal failure correlated with preservation of structural integrity as demonstrated by histological analyses in TSP-1 null mice, which showed significant reduction in the loss of brush border, cell necrosis, tubular dilation, cast formation, and vacuolization when compared with WT animals (Figure 6, B and C). Apoptosis in TSP-1 null mice was significantly reduced

TSP-1, a trimeric $450-\mathrm{kDa}$ glycoprotein, is a matricellular protein that interacts with several matrix components, cell receptors, soluble growth factors, and proteolytic enzymes (8, 15-17). TSP-1 belongs to the thrombospondin family of proteins and was initially recognized as a protein capable of inhibiting neovascularization induced by angiogenic factors (18). Subsequent studies localized the antiangiogenic region of TSP-1 to its procollagen-like region and the 3 properdin-like type 1 repeats (19). TSP- 1 can exert its antiangiogenic effect by inhibiting endothelial cell proliferation and migration $(20,21)$. CD36 has been implicated as an essential receptor for the proapoptotic, antiangiogenic effect of TSP-1, since studies have shown that TSP-1-mediated apoptosis is associated with the upregulation of active caspase- 3 and followed the binding to the transmembrane receptor $\operatorname{CD} 36(22,23)$. TSP-1 also induces endothelial cell apoptosis (8). TSP-1 expression in endothelial cells and in cancerous tissues is under the regulation of p53 (7); however, in kidney IR injury the expression of TSP-1 precedes the upregulation of p53 (Figure 2D), indicating that the pathways of activation of TSP may be different in the setting of ischemic injury. It is worth mentioning that, in addition to the kidney cortex (Figure 2C), NRK cells also express CD36 (data not shown). Interestingly, the expression of CD36 increased in kidney IR injury in a time course corresponding to the upregulation of TSP-1 (Figure 2C).
The proapoptotic activity of TSP-1 has been shown to be mediated via the activation of caspase- 3 and modulation of the $\mathrm{Bax} / \mathrm{Bcl} 2$ ratio (9). This relationship was verified in vitro by caspase- 3 enzymatic activity assay in cells exposed to TSP-1 (Figure 5C). In addition, TSP-1 is known to induce reorganization of the actin cytoskeleton and restructuring of focal adhesions through binding of TSP to a cell surface form of calreticulin (23). The background of broad functional aspects of TSP-1 increases its relevance as a protein expressed in acute IR injury, although these results have not been reported before in this setting. The only published reports of enhanced TSP-1 expression in kidney disease are limited to models of chronic interstitial damage associated with fibrosis (24-26). These studies suggest that TSP-1 may be an important promoter of TGF- $\beta$ activation, which in turn could influence chronic fibrosis in renal disease. Our data, however, do not support the temporal association between TSP- 1 and TGF- $\beta$ expression in the setting of kidney IR injury because the appearance of TGF- $\beta$ in IR injury is a much-delayed response (72 hours after IR injury) (27).

In the present report, TSP-1 expression in the setting of IR injury was predominantly localized to the portions of tubules that demonstrated morphological characteristics of cell injury (Figure 3), such as loss of brush border, cast formation, and tubular dilatation. In addition, the colocalization with the activated caspase- 3 in vivo clearly demonstrates that TSP-1 is expressed by cells undergoing apoptosis in the setting of IR. These findings are in support of the hypothesis that TSP-1 expression occurs rapidly in response to the induction of ischemic injury and that its expression is predominant in the parts of the tubules that sustain maximal injury. An important role for TSP-1 in mediating injury in proximal tubule cells is shown in NRK52 cells where addition of TSP-1 peptide resulted in cell damage as shown by cytochrome $c$ staining and caspase- 3 activation (Figure 5). While the addition of TSP-1 for 24-48 hours caused injury, it failed to do so after 3 hours of exposure in normal cultured kidney cells (Figure 5). Given the fact that in IR injury in vivo, kidney cells are oxidatively stressed, the experiments at 3 hours were repeated in cells subjected to ATP depletion, a model of in vitro IR injury. The results indicated significant caspase- 3 activation, consistent with the possibility that TSP-1 plays a detrimental role in causing injury in the setting of oxidative stress (Figure 5I).

The most salient features of the present studies are demonstration of the induction of TSP-1 in the setting of IR injury in kidney and the ability of TSP-1 to induce changes consistent with apoptosis in vitro, strongly indicating its role as not only a marker but also as a mediator of injury. The peak expression in the kidney occurred at 3 hours after reperfusion, well before the upregulation of $\mathrm{p} 53$, demonstrating that TSP- 1 activation in kidney injury is independent of p53. Morphologically, the most abundant expression of TSP-1 was observed in damaged tubules (predominantly proximal tubules) as a consequence of IR injury and showed colocalization with activated caspase-3. Demonstration of protection against ischemic renal failure and tissue damage in TSP-1 null mice (Figure 6) clearly indicates that the induction of TSP-1 in IR injury is detrimental and contributes to tubular damage and renal failure.

Contribution of the processes of necrosis and apoptosis to ischemia-induced acute renal failure is incompletely understood although both processes are known to occur as a result of the ischemic injury. Susceptibility to necrotic or apoptotic injury varies along the nephron depending upon the nature of injury, ongoing metabolic demands, and local oxygen availability. Apoptosis 
is an active, ATP/guanosine triphosphate-dependent process, and hence a rapid and abrupt depletion of ATP leads to necrosis as a predominant result of ischemic injury.

A cursory examination of apoptosis by TUNEL assay at 24 hours of reperfusion in kidneys of TSP-1 null mice and WT animals showed few TUNEL-positive cells in either group, which is not unexpected, given the published reports indicating the detection of apoptosis at an early phase (3-6 hours) of reperfusion, a time point very consistent with the detection of activated caspase- 3 in damaged tubules in kidneys of WT mice subjected to IR injury (Figure 4). However, a detailed examination of several fields from 3 WT and TSP- 1 null animals at 24 hours of IR injury showed significant reduction in the number of TUNEL-positive tubules in kidney cortices of TSP- 1 null animals. These results are in agreement with in vitro experiments (Figure 5) demonstrating the induction of apoptosis by TSP-1 in kidney tubule cells.

In summary, the present data identify TSP-1 as a novel protein identified in kidney ischemia. TSP-1 is induced in the early phase of IR injury, colocalizes with activated caspase- 3 in kidney nephrons, and induces proapoptotic changes in proximal tubule cells in vitro; its deletion in vivo provides protection against tissue injury and acute ischemic renal failure. We propose that TSP-1 is a mediator of injury and could be a novel therapeutic target for prevention of kidney damage in ischemic episodes.

\section{Methods}

Kidney IR injury: animal model. Kidney IR injury was induced in rats or mice according to established models and as previously described in detail (28-30). In brief, male Sprague Dawley rats (200-300 g) or CF57 black mice were anesthetized by intraperitoneal injection with pentobarbital sodium. The abdomen was shaved and prepped with Betadine solution and sterile drapes applied. Animals were placed on a heating pad to maintain constant temperature and monitored with a rectal thermometer. A midline abdominal incision was made, and the kidneys were exposed. The renal ischemia was induced by nontraumatic vascular clamps (Roboz Surgical Instrument Co.) over the pedicles for 30 minutes in rats and 45 minutes in mice. After the clamps were released, the incision was closed in 2 layers with 2-0 sutures. Sham-operated animals underwent anesthesia, laparotomy, and renal pedicle dissection only. All animals received warm saline instilled in the peritoneal cavity during the surgical procedure and were then allowed to recover with ad libitum access to food and water. At 3,12, 24, and 48 hours after ischemia, the animals were euthanized and their kidneys were harvested and snap frozen in liquid nitrogen or fixed in paraformaldehyde for immunohistochemical studies. All protocols involving animals were approved by the Institutional Animal Care and Use Committees of the University of Cincinnati and Johns Hopkins University. Blood samples were collected for measurement of serum creatinine.

RNA isolation. Total cellular RNA was extracted from mouse and rat kidney cortex and medulla according to established methods. In brief, $0.2-0.5 \mathrm{~g}$ of tissue was homogenized at room temperature in $10 \mathrm{ml}$ of Tri reagent (Molecular Research Center Inc.). RNA was quantitated by spectrophotometry and stored at $-80^{\circ} \mathrm{C}$.

DNA microarray. Poly(A+) RNA was extracted from the total RNA isolated from kidney cortices of 3 rats according to established protocols. cDNA labeled with ${ }^{33} \mathrm{P}$ was prepared using Superscript II (Life Technologies) using pooled poly $(\mathrm{A}+)$ RNA from each group $(2.5 \mu \mathrm{g}$ from each rat) and then used to probe 2 rat GeneFilters microarray containing 10,500 genes (Invitrogen Corp.). The same rat microarray filter was stripped and reprobed to compare both samples. Data were analyzed using Pathways software (Pathway2; Invitrogen Corp.) and normalized to multiple control points consisting of total genomic DNA. The nucleotide sequences of the genes or ESTs with the largest increases and decreases were subjected to basic local alignment with a BLAST search provided by the NCBI.

Northern bybridization. Total RNA samples were fractionated on a $1.2 \%$ agarose-formaldehyde gel. The samples were transferred to a nylon membrane, cross-linked by ultraviolet light and baked for 1 hour. Hybridization was performed according to established methods. The cDNA probes were labeled with ${ }^{32} \mathrm{P}$-deoxynucleotide, using the RadPrime DNA labeling kit (Invitrogen Corp.). Following hybridization, the membranes were washed, blotted dry, and exposed to a PhosphorImager cassette at room temperature for 24 to 48 hours, and read by PhosphorImager (Molecular Dynamics).

A ${ }^{32}$ P-labeled cDNA fragment of the mRNA-encoding TSP-1 (corresponding to nucleotides 3,790 to 4,415 of a mouse cDNA; GenBank accession number NM_011580) or p53 (corresponding to nucleotides 110 to 860 of a mouse cDNA; GenBank accession number AB020317) was used as specific probe.

Western blot analysis. Proteins were extracted from kidneys and subjected to Western blot analysis as described (29). Briefly, kidneys from mice subjected to 45 minutes of ischemia and 3 hours of reperfusion were homogenized in ice-cold isolation solution $(250 \mathrm{mM}$ sucrose and $10 \mathrm{mM}$ triethanolamine, $\mathrm{pH}$ 7.6) containing protease inhibitors (phenazine methyl sulfonyl fluoride, $0.1 \mathrm{mg} / \mathrm{ml}$; leupeptine, $1 \mu \mathrm{g} / \mathrm{ml}$ ). The homogenate was centrifuged at low speed $(1,000 \mathrm{~g})$ for 10 minutes at $4{ }^{\circ} \mathrm{C}$ to remove nuclei and cell debris. Proteins were resolved by SDSPAGE $(50 \mu \mathrm{g} / \mathrm{lane})$ and transferred to nitrocellulose membrane. The membrane was blocked with $5 \%$ milk proteins and then incubated for 6 hours with the TSP-1 antibody at 1:400 dilution. The sites of antigenantibody complex were visualized using chemiluminescence method (SuperSignal Substrate; Pierce Biotechnology Inc.) and captured on light-sensitive imaging film (Kodak).

Single- and double-immunofluorescence labeling. Paraformaldehyde-fixed paraffin-embedded sections from mouse kidney were washed twice in PBS (pH 7.4) and blocked with 10\% BSA/0.3\% Triton X-100 in PBS for 1 hour. Single- and double-immunofluorescence labeling with anti-TSP-1 antibodies was performed in our laboratories as described in ref. 28. The mouse monoclonal anti-TSP-1 antibody (Neomarkers; Lab Vision Corp.) detects the collagen type- $\mathrm{V}$ binding domain as the epitope, and its binding to TSP was unaffected by glucoseaminoglycans, hyluronic acid, or heparin. For double labeling, tissue sections were incubated with mouse anti-TSP monoclonal and rabbit anti-activated caspase-3 polyclonal (Sigma-Aldrich) antibodies. Sections were washed and then incubated with appropriate secondary antibodies (rabbit anti-goat IgG conjugated with Oregon green 488 for antiactivated caspase- 3 and goat anti-mouse IgG conjugated with Alexa Fluor 568 dye for anti-TSP-1) for 2 hours at room temperature. Sections were examined and images acquired on a Nikon PCM 2000 laser confocal scanning microscope as $0.5-\mu \mathrm{m}$ "optical sections" of the stained cells. The 488-nm line of the Argon laser, isolated with the standard Argon laser exciter filter supplied with PCM 2000, was used for the green dye excitation. The PCM 2000 standard 515/30 nm emission filter was used for the green-emitting dye. Red dye was excited with the 543.5-nm single-line output of the HeNe laser. The PCM 2000 standard red channel long pass $565-\mathrm{nm}$ filter was used as the emission filter for the red dye. Digital images of the green and red dyes were simultaneously acquired through a single illumination and detection pinhole. The images were discretely resolved into 2 channels and separately analyzed. All experiments (Northern hybridization, Western blotting and immunostaining) were performed in 3 to 5 animals at each time point.

RT-PCR of CD36 in mouse kidney. Based on the CDNA sequence of mouse CD36 (GenBank accession number BC010262), the following oligonucle- 
otide primers were synthesized and utilized for RT-PCR on RNA isolated from mouse kidney: 5'-ATGACGTGGCAAAGAACAGCAGC (sense), and 5 '-GCAACAAACATCACCACTCCAATCC (antisense). The cycling parameters were as follows: $94^{\circ} \mathrm{C}$ for 1 minute, then $94^{\circ} \mathrm{C}$ for 30 seconds, and $68^{\circ} \mathrm{C}$ for 3 minutes, 35 cycles each. The mouse PCR product was purified and used as probe for Northern hybridization.

Effect of TSP-1 on kidney cells in vitro. TSP-1 at $1 \mu \mathrm{g} / \mathrm{ml}$ was added to cultured kidney proximal tubule (NRK) cells and studied at 24 and 48 hours for evidence of apoptosis by cytochrome $c$ release and caspase- 3 activity. The NRK-52E normal kidney cell line is derived from the proximal tubule epithelium (PTE) of the rat kidney, is nontumorigenic, retains many morphological and physiological characteristics of PTE, and has been used to mimic changes in vivo (31-33). Cytochrome $c$ apoptosis detection kit (Alexa Fluor 488 assay; Invitrogen Corp.) was used to perform the apoptosis assay. In brief, cells were washed with $\times 1 \mathrm{PBS}$ at $37^{\circ} \mathrm{C}$ and fixed with a fixative solution. Cells were then permeabilized and prepared for cytochrome $c$ labeling. Immunocytochemical labeling was performed by using an anti-cytochrome $c$ mouse IgG in 1:500 dilution along with Alexa Fluor 488-goat anti-mouse IgG, a highly cross-adsorbed secondary antibody (1:1000 dilution). After washing, the slides were incubated with the nuclear dye Hoechst 33342 at the dilution 1:10,000 for 20 minutes. The slides were analyzed by confocal microscopy.

Caspase-3 colorimetric assay. Enzymatic activity of caspase- 3 was assayed in cultured cells by colorimetric assay according to manufacturer's protocol (R\&D Systems). For ATP depletion, cultured cells were incubated in PBS buffer twice, then incubated in KH buffer (see below) containing $7.5 \mu \mathrm{m}$ antimycin for 45 minutes, then washed with PBS 2 more times and changed back to normal media. $\mathrm{KH}$ Buffer comprised $118 \mathrm{mM} \mathrm{NaCl}$, $25 \mathrm{mM} \mathrm{NaHCO}_{3}, 4.8 \mathrm{mM} \mathrm{KCl}, 1.2 \mathrm{mM} \mathrm{MgSO}_{4}, 1.2 \mathrm{mM} \mathrm{KH}_{2} \mathrm{PO}_{4}$, and $2.5 \mathrm{mM} \mathrm{CaCl}_{2}$, adjusted to $\mathrm{pH}$ 7.4.

IR injury in TSP-1 null mice. WT and TSP-1 null mice (generous gifts from Jack Lawler, Department of Pathology, Harvard Medical School, Boston, Massachusetts, USA; ref. 34) were subjected to 45 minutes of ischemia and 24 hours of reperfusion as above ( $n=5$ for each group). Thereafter, animals were sacrificed, kidneys were removed, and blood was collected and processed for creatinine measurement.

TUNEL assay. Apoptosis in kidney sections was determined by TUNEL assay using the ApopTag in situ apoptosis detection kit (Chemicon International). The slides were counterstained with methyl green. A minimum of 10 fields per section, with 3 sections each from WT animals with IR injury, TSP-1 null animals with IR injury, and sham-operated WT or TSP-1 null animals were examined. Only tubules that contained TUNEL-positive cells with the characteristics of nuclear morphology of apoptosis including nuclear fragmentation and nuclear condensation by TUNEL assay were counted as apoptotic tubules. For statistical analysis, tubules with a minimum of 1 TUNEL-positive cell were counted as positive. The number of TUNEL-positive tubules was counted, and the results were expressed as TUNEL-positive tubules/field.

Histological analysis of kidneys in WT and TSP-1 null mice after sham surgery or IR injury. At 24 hours, the kidneys were removed and fixed in paraformaldehyde for 48 hours. Kidney sections were paraffin embedded and $5-\mu \mathrm{m}$ sections were stained with H\&E. Three sections each from WT animals with IR injury, TSP-1 null animals with IR injury, and sham-operated WT or TSP-1 null animals were examined for tubular dilatation, cast formation, loss of brush border, necrosis, and epithelial vacuolization. Six fields per section were examined. The morphologic changes were scored semiquantitatively on a 0 to $4+$ scale $(0$, no lesion; $1+,<25 \%$ of parenchyma affected by the lesion; $2+, 25-50 \%$ of parenchyma affected by the lesion; $3+$, $>50-75 \%$ of parenchyma affected by the lesion; and 4, $>75 \%$ of parenchyma affected by the lesion). The scores were added up and divided by the number of fields to determine the average score per field.

Statistics. Values are expressed as mean \pm SEM. The significance of difference between mean values was examined using ANOVA. $P<0.05$ was considered statistically significant.

\section{Acknowledgments}

These studies were supported by a Merit Review Award (M. Soleimani), NIH grants DK 66589 (M. Soleimani) and DK 54770 (H. Rabb), and grants from Dialysis Clinic Inc. (M. Soleimani).

Received for publication April 26, 2005, and accepted in revised form September 13, 2005.

Address correspondence to: Manoocher Soleimani, Division of Nephrology and Hypertension, Department of Medicine, University of Cincinnati, 231 Albert Sabin Way, MSB 259G, Cincinnati, Ohio 45267-0585, USA. Phone: (513) 558-5471; Fax: (513) 5584309; E-mail: Manoocher.soleimani@uc.edu.
1. Bonventre, J.V. 1993. Mechanisms of ischemic acute renal failure. Kidney Int. 43:1160-1178.

2. Tritto, I., et al. 1998. A short burst of oxygen radicals at reflow induces sustained release of oxidized glutathione from postischemic hearts. Free Radic. Biol. Med. 24:290-297.

3. Sutton, T.A., and Molitoris, B.A. 1998. Mechanisms of cellular injury in ischemic acute renal failure. Semin. Nephrol. 18:490-497.

4. Rabb, H., Wang, Z., Postler, G., and Soleimani, M. 2000. Possible molecular basis for changes in potassium handling in acute renal failure. Am. J. Kidney Dis. 35:871-877.

5. Sheridan, A.M., and Bonventre, J.V. 2000. Cell biology and molecular mechanisms of injury in ischemic acute renal failure. Curr. Opin. Nephrol. Hypertens. 9:427-434.

6. Safirstein, R.L. 2004. Acute renal failure: from renal physiology to the renal transcriptome. Kidney Int. Suppl. 66:S62-S66.

7. Dameron, K.M., Volpert, O.V., Tainsky, M.A., and Bouck, N. 1994. Control of angiogenesis in fibroblasts by p 53 regulation of thrombospondin-1. Science. 265:1582-1584.

8. Lawler, J. 2000. The functions of thrombospondin-1 and -2. Curr. Opin. Cell Biol. 12:634-640.
9. Nor, J.E., et al. 2000. Thrombospondin-1 induces endothelial cell apoptosis and inhibits angiogenesis by activating the caspase death pathway. J. Vasc. Res. 37:209-218.

10. Ratziu, V., et al. 1998. Zf9, a Kruppel-like transcription factor up-regulated in vivo during early hepatic fibrosis. Proc. Natl. Acad. Sci. U. S. A. 95:9500-9505.

11. Kaushal, G.P., Singh, A.B., and Shah, S.V. 1998. Identification of gene family of caspases in rat kidney and altered expression in ischemia-reperfusion injury. Am. J. Physiol. Renal Physiol. 274:F587-F595.

12. Kunduzova, O.R., et al. 2003. Prevention of apoptotic and necrotic cell death, caspase- 3 activation, and renal dysfunction by melatonin after ischemia/reperfusion. FASEB J. 17:872-874.

13. Lee, W.K., Bork, U., Gholamrezaei, F., and Thevenod, F. 2005. Cd(2+)-induced cytochrome c release in apoptotic proximal tubule cells: role of mitochondrial permeability transition pore and $\mathrm{Ca}(2+)$ uniporter. Am. J. Physiol. Renal Physiol. 288:F27-F39.

14. Star, R.A. 1998. Treatment of acute renal failure. Kidney Int. 54:1817-1831.

15. Chen, H., Herndon, M.E., and Lawler, J. 2000. The cell biology of thrombospondin-1. Matrix Biol. 19:597-614.
16. Bornstein, P. 2001. Thrombospondins as matricellular modulators of cell function. J. Clin. Invest. 107:929-934

17. Bornstein, P. 1992. Thrombospondins: structure and regulation of expression [erratum 1993, 7:237]. FASEB J. 6:3290-3299.

18. Rastinejad, F., Polverini, P.J., and Bouck, N.P. 1989. Regulation of the activity of a new inhibitor of angiogenesis by a cancer suppressor gene. Cell. 56:345-355.

19. Good, D.J., et al. 1990. A tumor suppressor-dependent inhibitor of angiogenesis is immunologically and functionally indistinguishable from a fragment of thrombospondin. Proc. Natl. Acad. Sci. U. S. A. 87:6624-6628.

20. Sottile, J. 2004. Regulation of angiogenesis by extracellular matrix. Biochim. Biophys. Acta. 1654:13-22.

21. Streit, M., et al. 1999. Overexpression of thrombospondin-1 decreases angiogenesis and inhibits the growth of human cutaneous squamous cell carcinomas. Am. J. Pathol. 155:441-452.

22. Patey, M., et al. 1999. Immunohistochemical study of thrombospondin and its receptors alpha root of beta 3 and CD36 in normal thyroid and in thyroid tumours. J. Clin. Pathol. 52:895-900. 
23. Goicoechea, S., Pallero, M.A., Eggleton, P., Michalak, M., and Murphy-Ullrich, J.E. 2002. The antiadhesive activity of thrombospondin is mediated by the N-terminal domain of cell surface calreticulin. J. Biol. Chem. 277:37219-37228.

24. Hugo, C., Shankland, S.J., Pichler, R.H., Couser, W.G., and Johnson, R.J. 1998. Thrombospondin 1 precedes and predicts the development of tubulointerstitial fibrosis in glomerular disease in the rat. Kidney Int. 53:302-311.

25. Daniel, C., et al. 2004. Thrombospondin-1 is a major activator of TGF-beta in fibrotic renal disease in the rat in vivo. Kidney Int. 65:459-468.

26. Murphy-Ullrich, J.E., and Poczatek, M. 2000. Activation of latent TGF-beta by thrombospondin-1: mechanisms and physiology. Cytokine Growth Factor Rev. 11:59-69.

27. Spurgeon, K.R., Donohoe, D.L., and Basile, D.P.
2005. Transforming growth factor-beta in acute renal failure: receptor expression, effects on proliferation, cellularity, and vascularization after recovery from injury. Am. J. Physiol. Renal Physiol. 288:F568-F577.

28. Zahedi, K., et al. 2004. Identification of stathmin as a novel marker of cell proliferation in the recovery phase of acute ischemic renal failure. Am. J. Physiol. Cell Physiol. 286:C1203-C1211.

29. Zahedi, K., et al. 2003. Expression of SSAT, a novel biomarker of tubular cell damage, increases in kidney ischemia-reperfusion injury. Am. J. Physiol. Renal Physiol. 284:F1046-F1055.

30. Rabb, H., et al. 1994. Role of CD11a and CD11b in ischemic acute renal failure in rats. Am. J. Physiol. 267:F1052-F1058.

31. Chang, S.H., Phelps, P.C., Berezesky, I.K., Ebersberger, M.L., Jr., and Trump, B.F. 2000. Studies on the mechanisms and kinetics of apoptosis induced by microinjection of cytochrome $\mathrm{c}$ in rat kidney tubule epithelial cells (NRK-52E). Am. J. Pathol. 156:637-649

32. Best, C.J., et al. 1999. H-ras-transformed NRK-52E renal epithelial cells have altered growth, morphology, and cytoskeletal structure that correlates with renal cell carcinoma in vivo. In Vitro Cell. Dev. Biol. Anim. 35:205-214.

33. Phelps, P.C., et al. 1995. Studies on the mechanism of sulofenur and LY295501 toxicity: effect on the regulation of cytosolic calcium in relation to cytotoxicity in normal and tumorigenic rat kidney cell lines. Cancer Lett. 97:7-15.

34. Lawler, J., et al. 1998. Thrombospondin-1 is required for normal murine pulmonary homeostasis and its absence causes pneumonia. J. Clin. Invest. 101:982-992. 